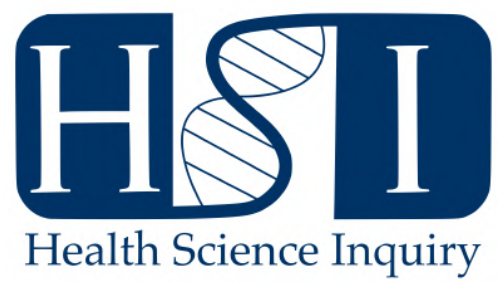

\title{
The Potential of Cannabis-derived Endocannabinoids as an Obesity Treatment
}

\author{
Hana Dibe ${ }^{1}$ \\ ${ }^{1}$ Human Health and Nutritional Science, University of Guelph
}

Before the recent legalization in Canada, marijuana was steadily the most widely used illicit drug [1]. With marijuana laws changing across North America, use is expected to rise and accordingly, while stigma against marijuana is shifting. Once consumed as an underground activity, marijuana is becoming widely accepted for its varying therapeutic benefits. Marijuana research has strong foundations, however, there is a lack of evidence-based research in humans which may pose a public health risk $[1,2,3]$. Derived from the Cannabis sativa plant, marijuana contains an abundance of different cannabinoids which interact with the human endocannabinoid system [4]. $\Delta^{9}$-tetrahydrocannabinol (THC) produces the sought-after psychoactive effects, however, the major non-psychoactive cannabinoid, cannabidiol (CBD), has new-found therapeutic potential [4]. CBD and THC have the same chemical formula; different structural arrangements give the two compounds completely different pharmacological properties (Figure 1) [4]. CBD has been demonstrated to have anxiolytic, anti-depressive, anti-convulsant and potential antidiabetic properties [5].

The endocannabinoid (EC) system is composed of endocannabinoids that bind to cannabinoid receptors in the central and peripheral nervous system [4]. The EC system controls food intake, energy balance and pain modulation, and may play a protective role in the development of Alzheimer's disease $[4,6]$. The two main G proteincoupled cannabinoid receptors, $\mathrm{CB} 1$ and $\mathrm{CB} 2$, primarily bind the endogenous cannabinoids anandamide (AEA) and 2-arachidonylglycerol (2-AG) [5]. CB1 is ubiquitously expressed in the brain, lungs, liver and kidneys, and CB2 is expressed in immune and hemopoietic cells [5]. While CB2 receptors are involved in mediating immunoregulatory actions, which play an important role in the inflammatory aspect of obesity, CB1 receptors (CB1R) centrally mediate appetite regulation, food intake and reward behaviours [5]. Interestingly, less recognized functions of peripheral CB1R in the liver, pancreas, skeletal muscle and adipose tissue include regulation of insulin signalling, glucose homeostasis, and hepatic lipogenesis [7].

With rates of obesity and type 2 diabetes (T2D) increasing across North America, research investigating new weight loss and insulin sensitizing treatments are vital. Due to its role in appetite regulation, the EC system has been researched as a potential target for obesity treatment. Plant endocannabinoids such as CBD are receiving attention as well $[4,5,6,8,9]$. In animal models of obesity, the EC system appears to be upregulated [4]. In three different rodent models of obesity, obese animals had higher hypothalamic levels of 2-AG that were correlated with increased food intake, suggesting that CB1R activation in the hypothalamus may increase food intake [4]. Wild type CB1R positive mice treated with HU210, a synthetic cannabinoid that acts on CB1R, experienced marked increases in hepatic mRNA expression of sterol regulatory element-binding protein 1 , acetyl-CoA carboxylase 1 and fatty acid synthase, suggesting upregulation of fat synthesis with CB1R activation [7].

Research using CB1R knockout (KO) animals supports the anti-obesity potential of CB1R antagonism. In a study examining whole body CB1R KOs, mice fed a high fat diet were protected from obesity, dyslipidemia, and leptin resistance $[7,10]$. Leptin is the fat-derived hormone that induces satiation and inhibits hunger following a meal. Leptin is elevated in obesity, likely a reflection of the increased fat mass, and leads to decreased sensitivity to the hormone, known as leptin resistance. Subsequently, reduced satiation often leads to overeating. In liver-specific CB1R KOs of the same study design, both insulin and leptin resistance were reduced, as well as steatosis, hyperglycemia and dyslipidemia. However, these mice were not resistant to weight gain, suggesting a central role for brain CB1R in regulating food intake [10].

Rimonabant was the first pharmacological CB1R blocker for obesity. In animal and human studies of obesity and T2D, rimonabant improved glucose homeostasis and caused significant weight loss. European markets approved rimonabant in 2006, however, in 2008, it was withdrawn worldwide due to serious psychiatric side effects, including suicide ideation [5]. Rimonabant may have interfered with normal CB1R function in the brain, explaining the psychiatric side effects. Both THC and CBD interact with CB1 and CB2 receptors, however, unlike endogenous cannabinoids, CBD is a partial antagonist a of CB1 receptors [8]. CBD is also a potent anti-inflammatory agent, shown to reduce levels of inflammatory cytokines IL- $1 \beta$ IL- 2 , IL- 6 , TNF- $\alpha$ and IFN$\gamma$ in a variety of animal studies [8]. Mice and humans share 


\section{Plant cannabinoids}
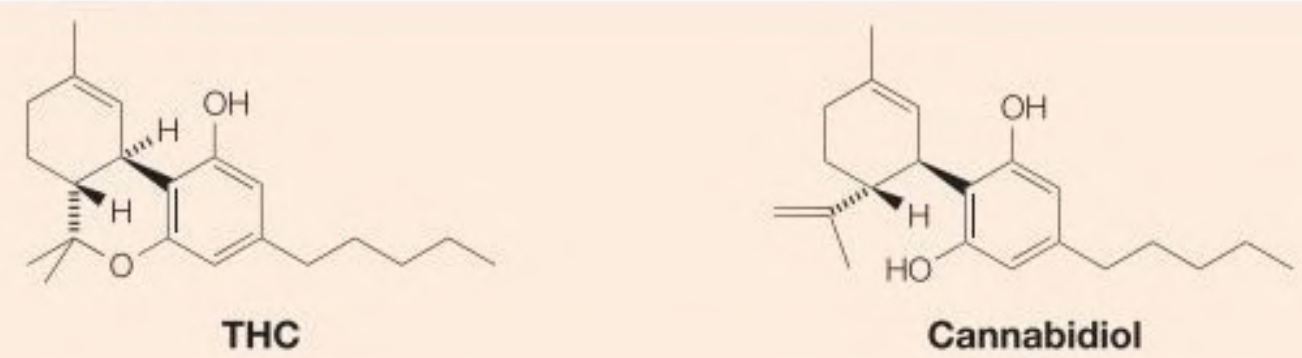

Figure 1: Chemical structure of plant endocannabinoids $\Delta^{9}$-tetrahydrocannabinol (THC) and cannabidiol (CBD). THC and CBD share the same chemical formula but where THC has a cyclic ring, CBD has a hydroxyl group $[4]$.

similar immune system make-up, therefore it is often accepted to use mouse models as a proxy for the low-grade chronic inflammation seen in obese humans [11]. In a clinical trial of T2D adults, $100 \mathrm{mg}$ of CBD oil taken orally twice daily for 13 weeks failed to elicit significant changes in fasting glucose and HOMA2 - a marker of $\beta$-cell function and insulin resistance. However, resistin levels, which are associated with insulin resistance, were significantly reduced, and GIP concentrations, a protein known to have pancreatic $\beta$-cell preserving effects, were increased [9].

$\mathrm{CBD}$ is a less potent antagonist than rimonabant. With its anxiolytic and anti-depressive properties, it is thought CBD will not cause the same side effects as rimonabant [9]. Further investigation of CBD and other similar cannabinoids in humans is warranted to better elucidate their potential role as an obesity treatment.

\section{REFERENCES}

[1] Rotermann M, Langlois K. Prevalence and correlates of marijuana use in Canada, 2012. Statistics Canada Ottawa, Ontario; 2015.

[2] Government of Canada. Canadian cannabis survey 2017 - Summary. Health Canada; 2017. Available from: https://www.canada.ca/en/healthcanada/services/publications/drugs-healthproducts/canadian-cannabis-survey-2017summary.html.

[3] National Academies of Sciences E, Medicine, et al. The health effects of cannabis and cannabinoids: The current state of evidence and recommendations for research. National Academies Press; 2017.

[4] Di Marzo V, Bifulco M, De Petrocellis L. The endocannabinoid system and its therapeutic exploitation. Nature reviews Drug discovery. 2004;3(9):771.

[5] Richey JM, Woolcott O. Re-visiting the endocannabinoid system and its therapeutic potential in obesity and associated diseases. Current diabetes reports. 2017;17(10):99.

[6] Iffland K, Grotenhermen F. An update on safety and side effects of cannabidiol: a review of clinical data and relevant animal studies. Cannabis and cannabinoid research. 2017;2(1):139-154.

[7] Osei-Hyiaman D, M D, Pacher P, Liu J, Radaeva S, Batkai S, et al. Endocannabinoid activation at hepatic CB1 receptors stimulates fatty acid synthesis and contributes to diet-induced obesity. J Clin Invest. 2005;115:1298-1305.

[8] McPartland JM, Duncan M, Di Marzo V, Pertwee RG. Are cannabidiol and $\Delta 9$-tetrahydrocannabivarin negative modulators of the endocannabinoid system? A systematic review. British journal of pharmacology. $2015 ; 172(3): 737-753$.

[9] Jadoon KA, Ratcliffe SH, Barrett DA, Thomas EL, Stott C, Bell JD, et al. Efficacy and safety of cannabidiol and tetrahydrocannabivarin on glycemic and lipid parameters in patients with type 2 diabetes: a randomized, double-blind, placebo-controlled, parallel group pilot study. Diabetes Care. 2016;39(10):1777-1786.

[10] Osei-Hyiaman D, Liu J, Zhou L, Godlewski G, HarveyWhite J, Jeong Wi, et al. Hepatic CB 1 receptor is required for development of diet-induced steatosis, dyslipidemia, and insulin and leptin resistance in mice. The Journal of clinical investigation. 2008;118(9):3160-3169.

[11] Della Vedova MC, Muñoz MD, Santillan LD, PlateoPignatari MG, Germanó MJ, Tosi MER, et al. A mouse model of diet-induced obesity resembling most features of human metabolic syndrome. Nutrition and metabolic insights. 2016;9:NMI-S32907. 


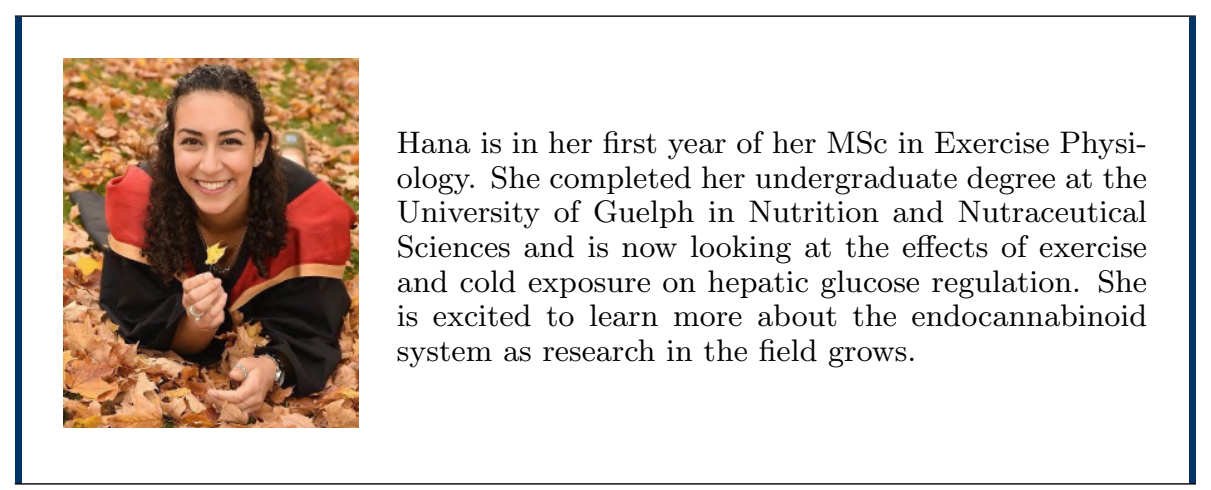

